\title{
Comparative Visualization of User Flows in Voice Portals
}

\author{
Björn Zimmer ${ }^{1}$, Dennie Ackermann ${ }^{1}$, Manfred Schröder ${ }^{2}$, \\ Andreas Kerren ${ }^{3}$, and Volker Ahlers ${ }^{1, \star}$ \\ 1 University of Applied Sciences and Arts Hannover, Faculty IV, \\ Dept. of Computer Science, P. O. Box 920251, 30441 Hannover, Germany \\ 2 HFN Medien GmbH, Ehlbeek 3, 30938 Burgwedel, Germany \\ 3 Linnæus University, School of Computer Science, Physics and Mathematics, \\ Vejdes Plats 7, 35195 Växjö, Sweden
}

\section{Introduction}

Voice portals are widely used to guide users interactively through an application. Recent portals provide a growing number of functions in one application, thus increasing their complexity. This work presents flow-map-based techniques for the comparative visualization of user flows at different time frames, in order to enable dialog designers to analyze and improve the user interaction with these systems.

Natural Language Systems in Voice Portals: More sophisticated voice portals use natural language systems (NLS), giving users the option to actually "talk" to the system in whole sentences. The system tries to interpret these sentences and interactively asks the user for detailed information, if necessary. Portals using NLS are rather large and complex, making it difficult to analyze their performance. Especially after applying changes to a voice portal or in case of technical problems, it is important to be able to analyze the consequences on user flows in the system.

\section{Comparing User Flows}

The user flow in a voice portal within a specific time frame corresponds to a weighted graph, where dialogs are represented by nodes and user flows by weighted edges. In order to compare user flows within the same voice portal at different time frames, a joined graph with multiple edges drawn as single, multi-colored arrows is created. The user value passing between two dialogs is visualized by varying either edge width or color saturation. The graph layout is based on a radial tree layout [2], which is manually adjusted. Two different approaches are discussed in the following.

* Contact author: volker.ahlers@fh-hannover.de

This work is financially supported by the German BMBF (grant no. 17N2809). 

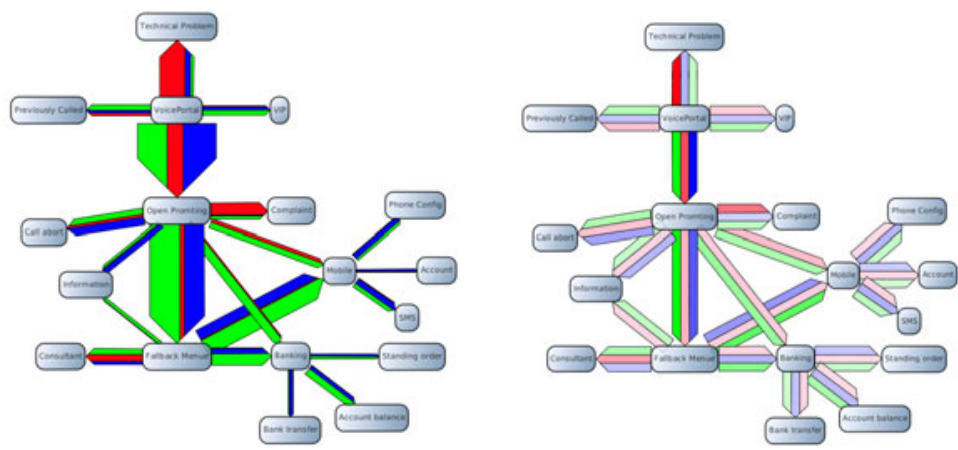

Fig. 1. Comparing user flows via edge width (left) and color saturation (right)

Varying Edge Width: Our first approach uses a flow map-based layout in such a way that the amount of users is represented by edge width, without merging edges that share the same direction [1]. Each color represents a different selectable time frame. The edge width represents the amount of users that have passed between the adjacent dialogs. The result for an example voice portal is shown in Fig 1 (left). The main drawback of this approach is that user flows between central dialogs of the portal lead to very thick edges. Peripheral edges are too thin to actually give a hint of the user flows. Very small flows are nearly invisible. To see the details at peripheral parts, the overall edge width could be increased, with the effect of making the graph look very crowded and unsettled. Additionally, viewing time frames with very different user flows could create the need to adjust node positions in order to route thick edges between them, destroying the mental map of the user [3].

Using Color Saturation: Representing user flows by color saturation avoids varying edge width. The result for the same example voice portal as used above is shown in Fig. 1 (right). Even smaller user flows at peripheral parts are now visible as low-saturated edges. A lower saturation threshold of 0.05 (for the range $[0,1])$ is used in order to keep colors distinguishable for small amounts of users. If no users have passed between two dialogs within a certain time frame, the corresponding color is not contained in the edge connecting these dialogs. Changing time frames does not have an impact on the edge width, thus preventing the need to adjust node positions.

\section{References}

1. Phan, D., Xiao, L., Yeh, R., Hanrahan, P., Winograd, T.: Flow Map Layout. In: InfoVis 2005, p. 29 (2005)

2. di Battista, G., Eades, P., Tamassia, R., Tollis, I.G.: Graph Drawing. Prentice Hall, Upper Saddle River (1999)

3. Eades, P., Lai, W., Misue, K., Sugiyama, K.: Preserving the Mental Map of a Diagram. Proc. of Compugraphics 91, 24-33 (1991) 